\title{
Spontaneous cervical haemorrhage of a parathyroid adenoma
}

\author{
Lara Ulrich', Graham Knee ${ }^{2}$ and Colin Todd ${ }^{3}$ \\ ${ }^{1}$ General Surgery ${ }^{2}$ Pathology ${ }^{3}$ Radiology, Kingston Hospital, London, UK
}

Correspondence

should be addressed

to L Ulrich

Email

lulrich1@doctors.org.uk

\section{Summary}

Haemorrhage of a parathyroid adenoma is a rare clinical presentation. This report describes a previously fit and well 54-yearold woman who presented with acute neck swelling and pain with an overlying ecchymosis. Admission laboratory tests revealed a raised parathyroid hormone and hypercalcaemia. A computed tomography (CT) scan showed widespread anterior cervical haemorrhage and a lesion at the inferior pole of the left thyroid gland. A working diagnosis of spontaneous haemorrhage from a parathyroid adenoma was made. As she was haemodynamically stable, she was treated conservatively with a period of observation in hospital to monitor for signs of neck organ compression. Follow-up imaging with $\mathrm{CT}$, ultrasound and sestamibi confirmed the likely source of haemorrhage as a parathyroid nodule with significant vascularity. The diagnosis was confirmed on histopathological analysis after elective surgical exploration of the neck 6 months after her presentation. This revealed a benign parathyroid adenoma with evidence of acute and chronic bleeding. The patient made a full recovery with immediate normalisation of her biochemistry post-operatively. Despite developing a hoarse voice in the immediate post-operative period, this resolved completely within 1 month. This case report provides further evidence to support a minimal delay for elective surgery after conservative management to reduce the risks associated with recurrent bleeding.

\section{Learning points:}

- Haemorrhage of a parathyroid adenoma should be a differential for all cases of acute cervical swelling or ecchymosis with no precipitating factor.

- The clerking should identify any risk factors for endocrine disease.

- Blood tests to screen for abnormal parathyroid biochemistry should be performed on admission.

- Detailed imaging of the neck is essential to identify the source of haemorrhage and risk of compression to vital neck organs.

- Conservative management is a suitable option for patients who remain haemodynamically stable but all should undergo a period of observation in hospital.

- Conservatively managed patients should be considered for definitive surgical exploration within a month of presentation to avoid the risks of recurrent bleeding.

\section{Background}

Spontaneous extracapsular haemorrhage of a parathyroid adenoma without a precipitating factor is a very unusual clinical presentation. Thirty similar cases have been reported in the literature, the first of which was identified on autopsy by Capps (1) after a fatal parathyroid haemorrhage. The prevailing theory to explain this 
manifestation is rapid growth of the parathyroid tumour which overwhelms its vascular supply (2). The parathyroid glands have a variable vasculature but usually receive the majority of their blood supply from branches of the inferior thyroid artery, occasionally supplemented by the superior thyroid artery. Whereas rupture of a thyroid nodule usually leads to intracapsular haemorrhage, rupture of a parathyroid lesion more commonly causes an extracapsular haemorrhage with extension of blood into the cervical subcutaneous tissues; this is secondary to its relatively thinner capsule (3). Consequently, localised symptoms of parathyroid haemorrhage include ecchymosis, diffuse neck swelling and tenderness.

The clinical presentation can vary more widely depending upon the extent of haemorrhage; tracking of blood into the mediastinum may manifest as chest pain, dyspnoea, cough and haemoptysis (4). There is also the potential for the expanding haematoma to compress neighbouring structures, leading to dysphagia and airway compromise (3) (4). In any patient presenting with spontaneous cervical haemorrhage, differential diagnoses such as dissecting aortic aneurysm, dissecting necrotising mediastinitis, atypical extracapsular haemorrhage of a thyroid nodule and iatrogenic trauma to the great vessels should be considered (2) (3). As this presentation of a parathyroid adenoma is so rare, each patient is presently treated on a case by case basis. Some studies have proposed diagnostic criteria to improve diagnostic accuracy but there is currently no standardised pathway to manage these patients (5).

\section{Case presentation}

A 54-year-old woman presented to the emergency department with a sudden onset rasping sensation in her throat, later accompanied by swelling and pain over the left side of her neck. She did not report any compressive symptoms such as dyspnoea or dysphagia, nor any systemic symptoms of inflammation or infection. This acute presentation was on a background of a 2-day history of progressive left ear ache. No triggering factors such as trauma, use of non-steroidal anti-inflammatories or anticoagulants were identified. Prior to this she was fit and well with no remarkable medical history or regular medications. Her mother and sister, however, both have known hypothyroidism for which they are on treatment with levothyroxine. On examination the left neck was diffusely swollen with overlying tenderness and an ecchymosis apparent beneath the skin.

\section{Investigation}

An initial computed tomography (CT) scan revealed extensive haemorrhage throughout the anterior portion of the neck causing right-sided tracheal deviation (Fig. 1). The ghost of a large nodule adjacent to the lower pole of the left lobe of the thyroid gland was noted as a possible source of cervical haemorrhage. Her admission blood tests showed a normal full blood count and liver function tests, corrected calcium of $2.76(n=2.20-2.60)$ parathyroid hormone (PTH) 19.3 ( $n=1.60-6.90)$, thyroid-stimulating hormone $1.7(n=0.27-4.20)$ and free thyroxine 13.6 $(n=9.0-25.0)$. Despite the findings of primary hyperparathyroidism and hypercalcaemia, she had not reported any major symptoms of raised calcium prior to admission.

\section{Treatment}

The patient was given a working diagnosis of spontaneous cervical haemorrhage of a vascular left parathyroid adenoma. She remained in hospital for 3 days for conservative treatment and continued observation. As she remained clinically and biochemically stable she was not a candidate for immediate surgical intervention. The patient was therefore discharged to be followed up in endocrine clinic with the hope that the parathyroid adenoma would self-infarct, also known as an autoparathyroidectomy.

\section{Outcome and follow-up}

A follow-up CT scan carried out 2 months after admission showed complete resolution of the cervical haemorrhage noted initially. A $17 \mathrm{~mm}$ well defined highly vascular nodule was identified in the same position, close to the lower pole of the left thyroid gland (Fig. 1). The aetiology of the mass remained unclear on imaging but remained

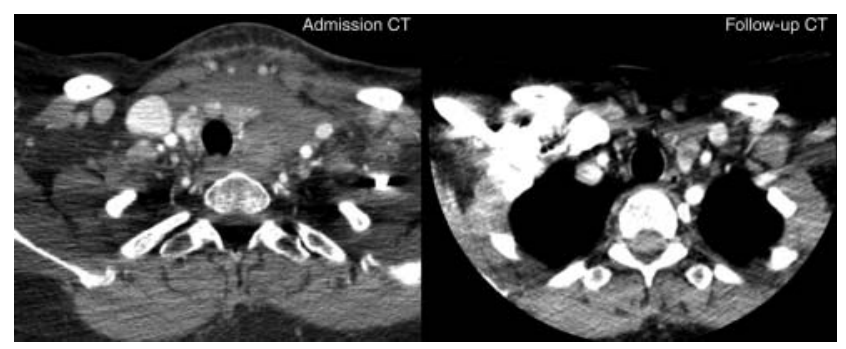

Figure 1

Admission CT scan showing extensive cervical haemorrhage with deviation of the trachea to the right. Follow-up CT scan showing resolution of haemorrhage and a left lower pole parathyroid adenoma. 


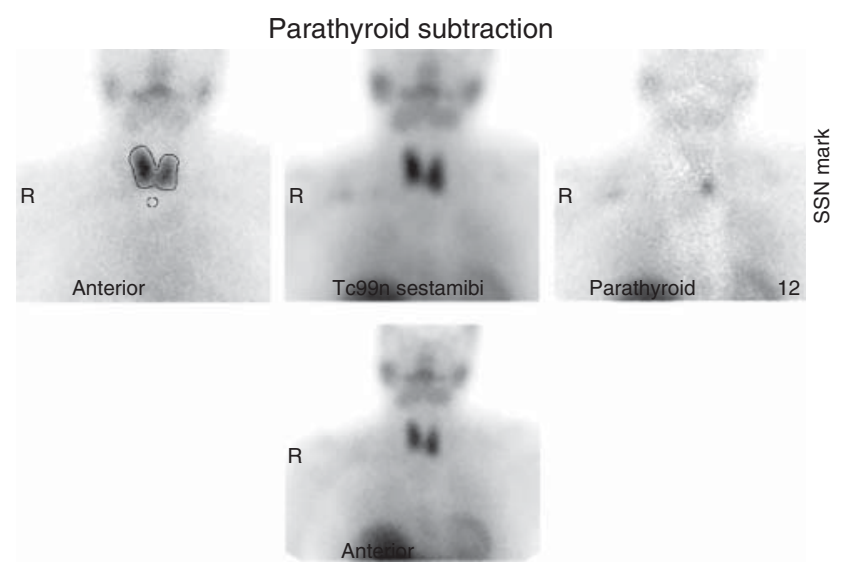

Figure 2

Subtraction sestamibi nuclear medicine scan showing a functional left lower pole parathyroid adenoma.

the likely source of spontaneous haemorrhage. The results of a second set of laboratory tests were as follows: corrected calcium 2.62 and PTH 21.1. Clinically, she reported an abnormal sensation within the throat but was otherwise asymptomatic. Indirect laryngoscopy revealed no abnormality within the larynx and the only finding on external examination was evidence of residual bruising over the left neck.

Parathyroid scintigraphy was arranged to confirm the nature of the vascular lesion noted on CT. This demonstrated a marked area of retained activity in relation to the lower pole of the left lobe of the thyroid, entirely consistent with the presence of a parathyroid adenoma (Fig. 2). An ultrasound scan (US) of the neck showed a slightly centric hypoechoic solid soft tissue mass in the lower pole of the left lobe of the thyroid gland with significant intra-lesional blood flow (Fig. 3). These findings were entirely congruent with the previous CT and scintigraphy scans which suggested a diagnosis of parathyroid adenoma measuring $1.8 \mathrm{~cm}$ in the widest axis. As the size of the nodule measured at just below $2 \mathrm{~cm}$, it was impossible to exclude a malignancy on imaging alone. In order to exclude the possibility of a parathyroid carcinoma and treat her hypercalcaemia, she was referred for elective surgery.

Elective surgical exploration of the neck revealed a fixed mass at the infero-posterior pole of the left thyroid gland which was attached to the inferior thyroid artery and left recurrent laryngeal nerve (RLN) posteriorly. This mass was dissected from an area of surrounding haemorrhagic change which included blood clot and fibrinous material. Histopathology findings confirmed encapsulated parathyroid tissue consistent with an adenoma, accompanied by evidence of both chronic and acute intraparenchymal haemorrhage (Figs 4 and 5). A third set of laboratory tests post-operatively revealed normalisation of serum adjusted calcium levels (2.33). Clinically, the patient's voice was hoarse following the surgery. In this case, it was likely secondary to the effects of the diathermy electricity in close proximity to the tethered RLN. At the post-operative follow-up clinic appointment her hoarse voice had completely resolved and her serum calcium remained within the normal range.

\section{Discussion}

A review of the literature shows that this case of parathyroid haemorrhage possesses many similarities to other case reports with regard to the patient demographic, clinical presentation, investigations performed and success of surgical intervention as definitive management. The majority of cases are seen in middle-aged females who are previously fit and well who present with predominantly localised symptoms which include acute cervical pain, swelling, tenderness and ecchymosis. None have reported an identifiable trigger such as trauma or anticoagulant therapy. The development of respiratory complications from mediastinal extension or compressive symptoms is only described in a few cases, with the latter requiring emergency surgery (2) (6).

In all cases where bloods tests have been performed on admission, almost every patient's biochemistry includes a degree of hypercalcaemia and hyperparathyroidism. This is most likely explained by rapid growth of the

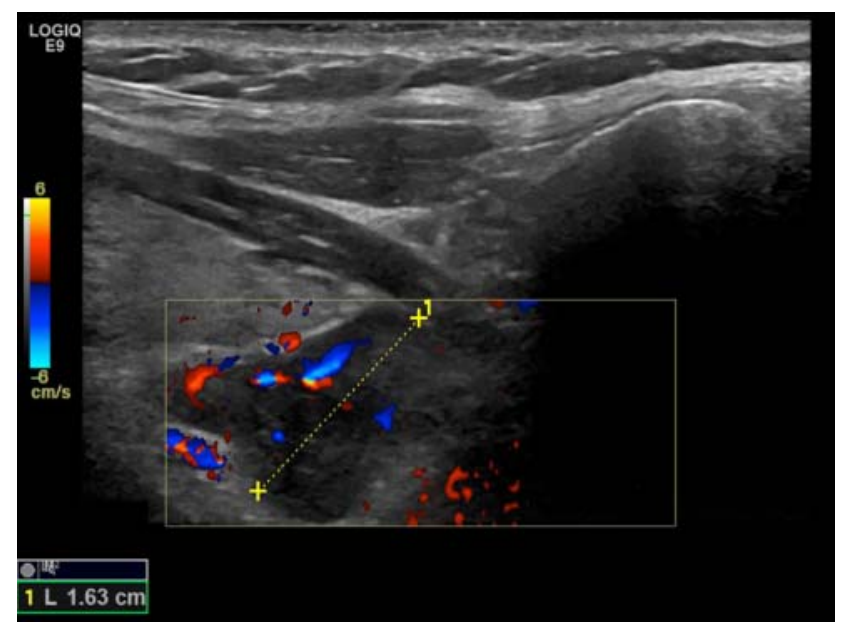

Figure 3

Ultrasound scan of the neck confirming a parathyroid adenoma. 


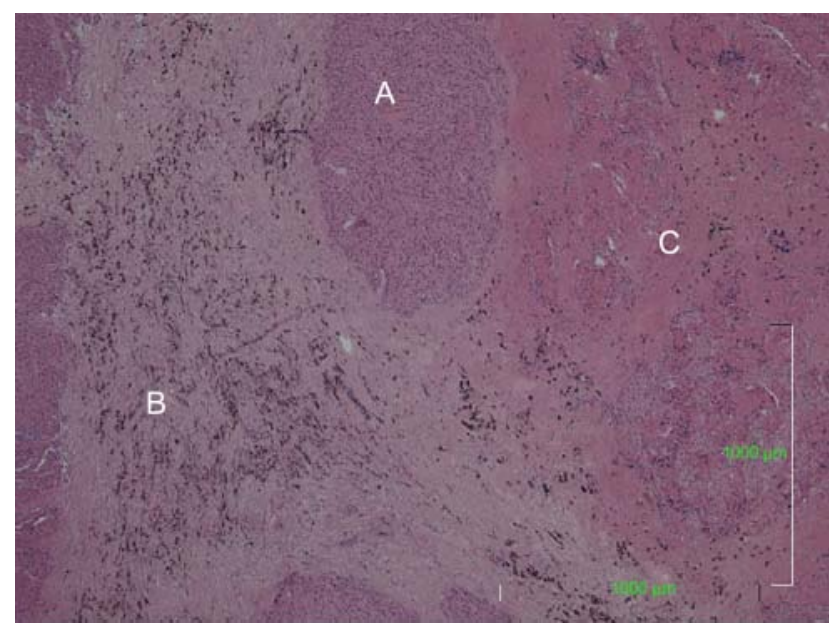

Figure 4

Histopathology specimen showing normal parathyroid tissue (A), haemosiderin deposition indicating chronic old haemorrhage (B) and fibrin deposition indicating more recent haemorrhage (C).

parathyroid adenoma leading to a larger volume of chief cells which secrete high amounts of PTH into the bloodstream. The effect of increased bone resorption and calcium reabsorption in the kidney causes hypercalcaemia. Studies have suggested that it is this rapid growth which eventually leads to an acute haemorrhage when demand outstrips the vascular supply. Shim et al. (7), however, reports a non-functional adenoma which could be explained by either tissue necrosis secondary to cystic degeneration or pressure from the haematoma compressing surrounding blood flow.

This case did present some new challenges to diagnosis and management not well recounted by other case reports. Our patient presented with a 2-day history of preceding left ear ache before the onset of acute symptoms. This may have been the consequence of referred cervical pain from the haematoma or indicate a possible viral trigger for this presentation. There were, however, no systemic signs of infection or inflammation. Unlike other reports, this patient had a strong family history of thyroid disease. While this is unlikely to be a risk factor for the manner in which a parathyroid lesion presents, identification of this family history at clerking might help speed up the diagnosis.

The most striking differences though were the 6 months delay between presentation and elective surgery in addition to the histological findings seen in Figs 4 and 5. In other cases where conservative management is used initially, definitive elective surgical intervention is usually performed within a month of presentation (3) (4).
The findings of acute and chronic haemorrhage within the parathyroid gland confirm that delayed elective management does not eliminate the risk of recurrent haemorrhage (6). This continued haemorrhage could explain the finding of a heavily tethered left RLN seen on surgical exploration. This made the surgery more difficult and led to temporary hoarseness after surgery. There is no collective decision on when to carry out elective surgery but if the delay risks recurrent bleeding and damage to the RLN, perhaps the recommendation should be within 1 month of presentation. To help identify those at risk of vocal cord palsies, indirect laryngoscopy should be performed on admission as an obligatory investigation to identify cord paresis deteoriation (6).

The indications for emergency surgery include acute anaemia, hypercalcaemic crisis, airway obstruction and hypotensive decompensation (3) (4). These complications may not arise immediately and this necessitates a period of monitoring in hospital. Ilicheva (6) describes a cyclical nature of presentation where acute localised symptoms were proceeded by a recovery period before later decompensating to neck organ compression. As this condition has the potential for delayed deteoriation, patients should be monitored in hospital initially with regular blood tests to identify an evolving anaemia or hypercalcaemic crisis. Once biochemically and clinically stable, these patients should be educated on discharge about warning symptoms of delayed decompensation such as swallowing and breathing difficulties.

In almost every case of parathyroid haemorrhage a CT and US of the neck have been ordered to quantify the extent of haemorrhage and establish the source of

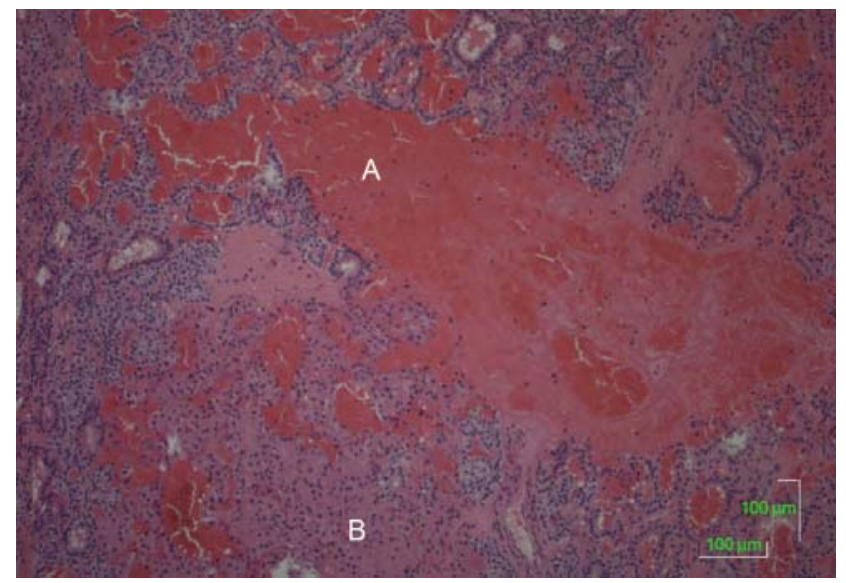

\section{Figure 5}

Histopathology specimen showing normal parathyroid tissue containing both oxiphilic and chief cells (B) with evidence of acute haemorrhage (A). 
bleeding. Scintigraphy is less widely used and in cases where the parathyroid sestamibi scan has been available, interestingly, the majority have not shown increased uptake in the area of concern. (2) (3) (6) (7). In our case, scintigraphy was a useful adjunct to confirming the diagnosis of parathyroid adenoma as there was a clear area of increased uptake. However, if the majority are negative, imaging with CT and US should suffice in diagnostic work up. The decision to image the chest for thoracic complications varies between cases. As mediastinal extension of the haemorrhage is rare, a logical recommendation would be to order plain film chest radiography only when symptoms of chest pain, dyspnoea and cough are present.

This case emphasises the importance of a full diagnostic work up for patients who present with acute neck swelling, pain or ecchymosis. This includes a thorough endocrine history, examination for associated complications of neck organ compression and laboratory investigations which include calcium and PTH levels. Detailed cervical imaging with CT and ultrasound will identify the source and extent of haemorrhage. While patients with acute neck organ decompression, anaemia or hypercalcaemic crisis should be considered for emergency surgery, stable patients can be managed conservatively with observation. To minimise the risk of recurrent bleeding and secondary damage to the RLN, definitive elective surgery should be performed without unnecessary delay.

\section{Declaration of interest}

The authors declare that there is no conflict of interest that could be perceived as prejudicing the impartiality of the case report.

\section{Funding}

This case report did not receive any specific grant from any funding agency in the public, commercial or not-for-profit sector.

\section{Patient consent}

Written consent has been obtained from the patient for publication of the submitted article and accompanying images.

Author contribution statement

L Ulrich wrote and submitted the article. G Knee provided the histopathological images and C Todd provided the radiological images.

\section{References}

1 Capps R 1934 Multiple parathyroid tumors with massive mediastinal and subcutaneous haemorrhage. American Journal of the Medical Sciences 188 800-805. (doi:10.1097/00000441-193412000-00007)

2 Yoshimura N, Mukaida H, Mimura T, Iwata K, Amioka A, Hirabayashi N \& Takiyama W 2014 A case of an acute cervicomediastinal hematoma secondary to the spontaneous rupture of a parathyroid adenoma. Annals of Thoracic and Cardiovascular Surgery 20 (Supplement) 816-820. (doi:10.5761/atcs.cr.12.02060)

3 Rehman HU, Markovski M \& Khalifa A 2010 Spontaneous cervical hematoma associated with parathyroid adenoma. CMAJ: Canadian Medical Association Journal 182 E632. (doi:10.1503/cmaj.091167)

4 Huang J, Soskos A, Murad SM, Krawisz BR, Yale SH \& Urquhart AC 2012 Spontaneous haemorrhage of a parathyroid adenoma into the mediastinum. Endocrine Practice 18 e57-e60. (doi:10.4158/EP11329.CR)

5 Simcic KJ, McDermott MT, Crawford GJ, Marx WH, Ownbey JL \& Kidd GS 1989 Massive extracapsular hemorrhage from a parathyroid cyst. Archives of Surgery 124 1347-1350. (doi:10.1001/archsurg.1989. 01410110109023

6 Ilicheva E 2015 Spontaneous cervical-mediastinal haematoma caused by haemorrhage into parathyroid adenoma: a clinical case. International Journal of Surgery Case Reports 6C 214-2177. (doi:10.1001/archsurg.1989. 01410110109023)

7 Shim WS, Kim IK, Yoo SD \& Kim DH 2008 Non-functional parathyroid adenoma presenting as a massive cervical hematoma: a case report. Clinical and Experimental Otorhinolaryngology 1 46-48. (doi:10.3342/ceo. 2008.1.1.46)

Received in final form 26 April 2015

Accepted 28 April 2015 